\title{
Brine shrimp toxicity and invitro antimalarial activity of Citrus aurantifolia (Christm.) Swingle against Plasmodium falciparum 3D7
}

\author{
*Saroj Bapna, Mira Ramaiya and Abhay Chowdhary \\ Haffkine Institute for Training, Research and Testing,Acharya Donde Marge, Parel, Mumbai-400012, India
}

\begin{abstract}
Malaria is one of the most prevalent, devastating parasitic infectious diseases in the world. In an attempt to search for new antimalarial compounds efforts are being made to do preliminary screening of aerial parts of the plant Citrus aurantifolia (Christm.) Swingle (family Rutaceae) at early flowering and fruiting stages against chloroquine sensitive Plasmodium falciparum 3D7 strain. The plant extract were prepared in three extract forms: Ethyl acetate, Methanol and aqueous extract using standard procedure. The highest antimalarial activity was observed with ethyl acetate extract $\left(I C_{50} 42.73 \mu \mathrm{g} / \mathrm{mL}\right)$ as compared to methanolic extract $\left(I C_{50}\right.$ $94.18 \mu \mathrm{g} / \mathrm{mL})$ followed by aqueous extract $\left(I C_{50}>100 \mu \mathrm{g} / \mathrm{mL}\right)$. The toxicity of the extract was monitored with the brine shrimp lethality assay and showed no toxicity in all solvent extract tested. The preliminary phytochemical screening of aerial parts of the plant showed the presence of alkaloids, saponins, flavonoids and glycosides in all the three solvent extracts may contain antiplasmodial molecule that could be isolated by bio-guided phytochemical studies.
\end{abstract}

Keywords: Plasmodium falciparum, Citrus aurantifolia, antimalarial plants, Brine shrimp

\section{Introduction}

Malaria is one of the most severe public health problems worldwide though it is entirely preventable and treatable mosquito borne illness. It is a leading cause of death and disease in many developing countries, where young children and pregnant women are the groups most affected. According to the World health Organisation's World Malaria report 2013 and Global Malaria Action plan, 3.4 billion people (half of the world's population) live in areas of malaria transmission in 106 countries and territories [1]. In 2012, malaria caused an estimated 207 million clinical episodes, and 627,000 deaths.

The fight against malaria remains a constant challenge due to rising prevalence of Plasmodium falciparum resistance to chloroquine and many available drugs. New antimalarial agents are urgently needed to counter the spread of drug resistance malaria [2]. The long established use of quinine, artemisinin and its derivatives as highly effective antimalarial demonstrated that plant species are an important resource for the discovery of new antimalarial agents for prophylaxis and treatment. During last decade, several fundamental researches have been conducted to explore anti-malarial activity of many plants [3].

In traditional medicine whole plant or mixture of plants are used rather than isolated compounds. There are no records attesting the resistance to whole-plant extracts possible due to the synergistic action of their constituents; phytotherapy possible produces fewer adverse effects than chemotherapy [4].

In the present investigation aerial parts (a combination of different parts of the same plant) Citrus aurantifolia (Christm.) were tested against Plasmodium falciparum 3D7 strain by in vitro method. The plant is commonly known for its therapeutic effectiveness in a variety of diseases in traditional medicine. Furthermore, this plant is among the frequently used medicinal plant with antimalarial properties in Nigeria [5]. .Lime extract has an antimicrobial activity against upper respiratory tract bacterial pathogens [6]. The roots of $C$. aurantifolia used in traditional medicine for the treatment of fever. A wide range of bioactive ingredient from the citrus species has been found to exert anti-infection and anti-inflammatory activities [7]. The plant has shown antiallergic, anti-inflammatory, anti-cancer, anti-bacterial and anti-protozoal properties [8]. Several anti-oxidant products have also been identified in the seeds of Citrus plant [9]. The present investigation has been undertaken with an objective to establish phytochemicals, cytotoxicity and invitro anti-plasmodial activity of this plant, so that possible plant material could be explored for the treatment of malaria.

\subsection{Experimental}

\section{Material and Methods}

\subsubsection{Plant collection and Identification}

Aerial parts of the plant Citrus aurantifolia (Christm.) Swingle commonly known as lime was collected from Mumbai ( $\left.18^{\circ} 55^{\prime} \mathrm{N}, 72^{\circ} 54^{\prime} \mathrm{E}\right)$ India during May, 2012. The plant was identified by Dr. U. C. Bapat, 
Head, Department of Botany and Director, Blatter Herbarium, St.Xavier's College, Mumbai. The sample voucher specimen (R2399) of the plant was deposited in the Blatter herbarium.

\subsubsection{Extraction of plant material}

The Aerial parts of very young plant (secondary branches with tender and young leaves, flower buds, flowers and small unripe fruits) were separated manually and washed twice with distilled water, shade dried, powdered and extracted into 3 extract forms separately which consisted of ethyl acetate, methanol and aqueous using standard extraction procedures [10]. Solvents were removed under reduced pressure by using rotary vacuum evaporator. The per cent yield of extract in each solvent was determined and the crude extracts were stored at $4^{\circ} \mathrm{C}$ prior to antimalarial assay. Preliminary phytochemical screening was done using standard procedures [11].

\subsection{3. .Plasmodium falciparum culture and maintenance}

The Plasmodium falciparum 3D7 strain was procured from Indian Institute of Technology (IIT), Mumbai, India, was maintained in continuous culture by the modified method of Jensen and Trager [12] in O+ human red blood cells at a 5\% haematocrit in RPMI 1640 medium, supplemented with L-glutamine (4.2mM), HEPES $(25 \mathrm{mM}), \mathrm{NaHCO}_{3}(25 \mathrm{mM})$ hypoxanthine $(6.8 \mathrm{M}), 0.5 \%$ AlbumaxII (Invitrogen) and $50 \mu \mathrm{g} / \mathrm{ml}$ Gentamicin. Cultures were incubated at $37^{\circ} \mathrm{C}$ in an atmosphere of $5 \% \mathrm{CO}_{2}, 91 \% \mathrm{~N}_{2}$, and $3 \% \mathrm{O}_{2}$. Chloroquine was used as positive control.

\subsubsection{Invitro antiplasmodial assay}

The in vitro activity of crude leaf and stem bark extracts were evaluated against $P$. falciparum by means of the Mark III test, as developed by the WHO [13]. Briefly, the parasite cultures, prior to experimentation, were synchronized to the ring stage by treatment with 5\% D-sorbitol [14]. The two fold serial dilutions of the extracts were prepared in duplicate in 96 well flat bottomed micro plates. Solvent control of $0.5 \%$ DMSO and $0.1 \%$ methanol to check the effect of solvents on parasite maturation. Filter sterilized extracts $(100,50,25,12.5,6.25$ and $3.125 \mu \mathrm{g} / \mathrm{mL})$ were incorporated in 96 well tissue culture plate containing $200 \mu \mathrm{L}$ of P. falciparum culture with $2 \%$ haematocrit and $1 \%$ ring stage infected erythrocytes (parasitemia). The plates were incubated at $37^{\circ} \mathrm{C}$ in $\mathrm{CO}_{2}$ incubator for $40-48 \mathrm{~h}$. Parasitemia was evaluated after $48 \mathrm{hrs}$ by light microscopy using Giemsa-stained smears as described by Le Bras and Deloron [15].

The antiplasmodial activities of extracts were expressed by $\mathrm{IC}_{50}$, the inhibitory concentrations of the drug that induced 50\% reduction in parasitemia compared to control (100\% parasitemia).

\subsection{5 .Morphological changes in erythrocytes}

To assess morphological changes in erythrocytes attributed due to extract concentration erythrocytes were incubated with the highest concentration of the extract used in the antiplasmodial assay and maintained the same condition as in the case of antiplasmodial assay along with the control uninfected erythrocytes without extract. After $48 \mathrm{~h}$ of incubation, thin blood smears were stained with Giemsa stain and observed for morphological changes under microscope. The morphological findings were compared with control [16].

The preliminary phytochemical test was carried out using specific reagents through standard Procedure [17].

\subsubsection{Brine shrimp toxicity bioassay}

Inhibition of the growth of brine shrimps (Artemia salina) was used as a measure of the toxicity of the extract using brine shrimp lethality bioassay [18 ]. The eggs of the brine shrimp were collected from an aquarium shop (Mumbai, India) and hatched in artificial sea water (38g/lit saline) under constant aeration for 48 h. After hatching active nauplii free from egg shells were collected from brighter portion of the hatching chamber and used for the bioassay. Extracts were dissolved in DMSO and then serially diluted in artificial sea water to the desired concentrations. The final DMSO concentration did not exceed $1 \%$, which was shown not to have any harmful effect on the larvae. About $0.5 \mathrm{ml}$ of diluted test solution was added to the pre marked test tubes containing $4.5 \mathrm{ml}$ of artificial sea water. Finally 10 active shrimps were added into each test tube. A vial containing $50 \mu \mathrm{l}$ DMSO diluted to $5 \mathrm{ml}$ was used as control. Potassium permanganate (LC50 =5 $\mu \mathrm{g} / \mathrm{mL}$ ) was used as positive control, and vials containing shrimps, but without any extracts were included in each test. After $24 \mathrm{~h}$ the numbers of the survivors were counted using a magnifying glass and $\%$ mortality was calculated for each dilution as well as for control. The toxicity of each extract was determined from LC50 values (Lethal concentration of sample required to kill $50 \%$ of brine shrimp) in a set of three test tubes per dose. 


\section{Results}

The basic measurement of antimalarial activity used in this study was the reduction in number of parasitized erythrocytes in extract treated test cultures as compared to control (100\% parasitemia) without drug at $48 \mathrm{~h}$ of incubation period.

From the literature antimalarial activity of extracts was defined according to the IC50 values obtained. An extract showing an IC50 value $\leq 50 \mu \mathrm{g} / \mathrm{mL}$ was classified as active and extracts with activity beyond this range were considered in active [19].

Based on this classification, the ethyl acetate extract of Citrus aurantifolia (Christm) induced a significant decrease of parasite proliferation and showed promising antimalarial activity $\left(\mathrm{IC}_{50} \mathrm{Of} 42.73 \mu \mathrm{g} / \mathrm{mL}\right)$, as compared to methanolic ( $\mathrm{IC}_{50}$ of $94.18 \mu \mathrm{g} / \mathrm{mL}$ ). The aqueous extract $\left(\mathrm{IC}_{50}>100 \mu \mathrm{g} / \mathrm{mL}\right.$ ) was found inactive.

The microscopic observation of the uninfected erythrocytes incubated with the highest concentration of extract used in assay and normal erythrocytes without drug from the blank column of the 96 well plates showed no morphological difference after $48 \mathrm{~h}$ of incubation

The preliminary phytochemical screening of aerial parts of the plant $C$. aurantifolia showed the presence of alkaloids, saponins, flavonoids and glycosides in all the three extract form.

A brine shrimp cytotoxicity assay was used to predict the potential toxicity of the extracts. The selectivity index (SI) is defined as the ratio of the brine shrimp toxicity to the anti-plasmodial activity and is determined by dividing the $\mathrm{LC}_{50}$ values for the brine shrimp nauplii by the $\mathrm{IC}_{50}$ value for Plasmodium falciparum. The extract with higher selectivity (high SI value) indicate potentially safer therapy.

In brine shrimp lethality bioassay, $\%$ mortality increased gradually with increase in concentration of the test samples in comparison to positive control $\mathrm{KMnO}_{4}$. The toxicity potentiality exhibited by Citrus aurantifolia was very negligible or did not show any apparent toxicity as compared to control. The results suggest that all the extract of $C$. aurantifolia possesses considerable antiplasmodial activity and negligible cytotoxicity with selectivity index (SI value) ranged from 3.50 to 4.01

\section{Discussion}

Crude extracts are the simplest of available medications and are still promoted by WHO policies as emerging alternative systems of medicine to reach the large population not covered by formal medical care in remote areas. Developing countries, where malaria is endemic, depend strongly on traditional medicine as a source for inexpensive treatment of this disease [3]. The present study aids important impetus to the area of new antimalarial agents from plants source. Earlier claims show that the plant is used in traditional medicine and cultivated throughout India [20].

Different solvent extract of any particular plant held different antimalarial activity. The present study was investigated with the extract derived from aerial parts of the plant, Citrus aurantifolia (Christm). Out of the three extract form the ethyl acetate extract of this plant has shown highest antimalarial activity (IC 5042.73 $\mu \mathrm{g} / \mathrm{mL})$ as compared to methanol and aqueous extracts. This revealed that the active compounds are more soluble in non polar solvent as compared to methanol and water extract, and that changes the efficacy on parasites.

The antiplasmodial activity of this plant was previously reported showed antimalarial activity at higher concentration [19] in methanol extract. In the present investigations the ethyl acetate extract of aerial parts of very young plant at fruiting stage, demonstrated promising antimalarial activity. Importantly, many cases are known where the crude biological extract is more efficient pharmacologically than the most active purified compound from this extract. This could be due to synergism with other compounds present in the extract, which as such have no pharmacological activity [21]. It is also evident that none of the three solvent extract tested was toxic to the arthropod Artemia salina (Artemiidae) nauplii. This observation may be an indicator of their safety as drugs for mammalian organism. The results are summarized in table 1.

\section{Conclusion}

The present study provided evidence for the rational exploration of Indian medicinal plants as a source of antiplasmodial agents. The plant extracts however possess moderate antimalarial activity when compared with standard drug chloroquine. The results are encouraging and further work is needed for complete isolation, identification, and characterization to elucidate the active compound and their in-vivo anti-malarial efficacy in murine malaria model with comprehensive animal toxicity study.

\section{Acknowledgement}

The financial support of ICMR, New Delhi (Grant code: 59/39/2010/BMS/TRM) is gratefully acknowledged. 


\section{References:}

[1]. World Health Organization 2013. World Malaria Report. World health Organization,Geneva

[2]. C.W.Wright, Traditional antimalarials and development of novel antimalarial drugs. Journal of Ethnopharmacol 100, 2005 67-71.

[3]. S.Saxena, N. Pant, R.S. Bhakuni, , Antimalarial agents from plant sources. Cur. Sci.85 2003, 1314-1326.

[4]. M. L. Willcox and G. Bodeker. Traditional herbel medicine for malaria. BMJ, 329, 2004, 1156-9.

[5]. Taiye R Fasola, Pass C lyamah. Comaring the phytochemical composition of some plant parts commonly used in the treatment of malaria. Int. J. pure Appl. Sci Technol. 21 (1) 2014, 1-11

[6]. I..A. Adeleye, ,L. Iand Opieh, Antimicrobial activity of extract of local cough mixture on upper respiratory tract bacterial pathogens. West Indian Med. J. 52 (3), 2003, 188-190

[7]. A..Murakami, Y. Nakamura, Y. Ohto, Supressive effects of Citrus fruits on free radical generation and nobiletin, an antiinflamatorypolymethoxy flavonoids. 12, 2000,187-192

[8]. G. K.Jayaprakasha. K.K.Mandodi, S.M. Poulose, M, Jadegoud, B.S.Patil , Novel triterpenoid from Citrus aurantium L. possesses chemopreventive properties against human colon cancer cells. Biorganic Med. Chem., 16 (11), 2008,5939-5951

[9]. G.K.Jayaprakasha, and B.S. Patil Invitro evaluation of the antioxidant activities in fruit extract from citron. Food Chem., 101 (1), 2007, 410-418

[10]. C. K. Kokate Practical Pharmacognosy 2000. Vallabh Prakashan pp218

[11]. J. B. Harborne, Phytochemical methods-A Guide to Modern Techniques of Plant Analysis, Chapman and Hall, London.1998.

[12]. J. B. Jensen, W. Trager, Cultivation of erythrocytic and exoerythrocytic stages of Plasmodium in malaria. Academic Press. 2,1980, 280

[13]. WHO 2001. In vitro micro-test (Mark III) for the assessment of the response of Plasmodium falciparum to chloroquine, mefloquine, quinine, amodiaquine, sulfadoxine / pyrimetamine and artemisinin. Division of Control of Tropical Disease Review 2 CTD/MAL/97.20

[14]. C. Lambros, J. P.Vanderberg, Synchronization of Plasmodium falciparum erythrocytic stages in culture. Journal of Parasitology $65,1979,418-420$

[15]. J. Le Bras ,P. Deloron, In vitro study of drug sensitivity of Plasmodium falciparum: evaluation of a new semi-microtest. American Journal of Tropical Medicine and Hygiene 274, 1983,14218-14223

[16]. S. Ravi kumar, I. S.Jacob, P. Sughanti, Seaweeds as a source of lead cpmpounds for the decelopment of new antiplasmodial drug from South east Cost of India. Parasitol Res. 109, 2011 47-52. 5.

[17]. J. Parekh, R. Nair, S. Chanda, Preliminary screening of some folklore medicinal plants from Western India for potential antimicrobial activity. .Indian J. Pharmacology 37. 2005, 406-40920.

[18]. B. N. Meyer, N. R. Ferrigni, J. E. Putnam, L. B. Jacobsen, D. E. Nichols, J. L. McLaughlin. Brine shrimp: A convenient general bioassay for active plant constituents. Planta Med. 45, 1982, 31-34.

[19]. A. Ramazani, S. Zakeri, S. Sardari, N. Khodakarim, N. D.Djadidt, In vitro and in vivo anti-malarial activity of Boerhavia elegans and Solanum surattense. Malaria Journal, 9(124), 2010, 1-8

[20]. A. N. Awadh, Ali, K.Al-rahwi and U. Lindequist . Some medicinal plants used in Yemeni herbal medicine to treat Malaria. Afr. J. Trad. Cam. 1, 2004, 72-76. 18.

[21]. H Wagner and G. Ulrich-Merzenich, "Synergy research: approaching a new generation of phytopharmaceuticals," Phytomedicine, 16, (2-3), 2009, 97-110

Table 1. In vitro bioassays on Plasmodium falciparum 3D7 strains and toxicity assay of the Citrus aurantifolia.

\begin{tabular}{|l|l|l|l|l|l|}
\hline S. No. & Extract form & \%Extract yield & $\begin{array}{l}\text { Anti-plasmodial activity } \\
\mathrm{IC}_{50} \mu \mathrm{g} / \mathrm{mL}\end{array}$ & $\begin{array}{l}\text { Artemia salina } \\
\mathrm{LC}_{50} \mu \mathrm{g} / \mathrm{mL}\end{array}$ & Selectivity index (SI) \\
\hline 1. & Ethyl acetate & 3.87 & $42.73 \pm 3.23$ & $149.65 \pm 2.87$ & 3.50 \\
\hline 2. & Methanol & 7.65 & $94.18 \pm 2.91$ & $376.85 \pm 4.90$ & 4.01 \\
\hline 3. & Aqueous & 11.36 & $>100$ & $586.17 \pm 8.13$ & NA \\
\hline
\end{tabular}

IC50, the inhibitory concentration of extract that induced $50 \%$ reduction in parasitemia LC50: the lethal concentration that reduced the number of viable shrimp larvae by $50 \%$ Selectivity index $(\mathrm{SI})=$ Ratio LC50/IC50 